\title{
Investigation of surface roughness influence on hyperbolic metamaterial performance
}

\author{
Serge Kozik $^{1 *}$, Mohammed A Binhussain ${ }^{2}$, Andrei Smirnov ${ }^{1}$, Nikolai Khilo ${ }^{1}$, Vladimir Agabekov ${ }^{3}$ \\ ${ }^{1}$ B.I.Stepanov Institute of Physics, National Academy of Sciences of Belarus, Minsk, Belarus \\ ${ }^{2}$ The National Center for Building System KACST, Riyadh, Saudi Arabia \\ ${ }^{3}$ Institute of Chemistry of New Materials NASB, Minsk, Belarus \\ *corresponding author, E-mail: s. kozik@dragon.bas-net.by
}

\begin{abstract}
Light scattering on rough surface with nano-sized features needs simulation with rigorous electromagnetic solver. The performance of such grid method as FDTD is closely connected with mesh size of computational domain and curvilinear surface approximation. The main goal of this work was to introduce simple model of surface roughness which does not involve objects with complicated shapes and could help to reduce computational costs. We described and proved numerically that the influence of surface roughness at the interfaces in metal-dielectric composite materials could be described by proper selection of refractive index of dielectric layers. Our calculations show that this model works for roughness with RMS value about $1 \mathrm{~nm}$ and below. Some examples of roughness realization with narrow spatial spectrum and high LER value could not be described properly by simple dielectric index modification, thus forming the limits of this simple roughness model applicability.
\end{abstract}

\section{Introduction}

The necessary stage for every metamaterial engineering process is numerical simulation which predicts reflection, transmission spectra, effective refractive index and other measurable parameters depending on its structural design. Since plasmon resonances are highly sensitive to shape of structures and presence of closely situated objects it is very important to include imperfection of real structures in simulation model for better agreement with experimental performance of the samples [1]. Hyperbolic metamaterial behavior could be described by effective medium theory based on permittivity averaging. However, light scattering on rough surface with nano-sized features should be simulated with rigorous electromagnetic solver. We used finite-difference time-domain method (FDTD) with code written by the authors [2].

Modeling of surface roughness is closely connected with mesh size of computational domain and curvilinear surface approximation in FDTD. It is known that "staircace" approximation of complex shaped objects in rectangular grid is the most sufficient error source in FDTD calculation of dispersion materials. When dielectric materials are considered, there is effective technique of improving grid resolution by averaging of material parameters over a unit grid cell [3]. Some works show that smoothing is possible for plasmonic problems with flat interfaces [4], for 2D problems [5] and for the case of simple Drude model of permittivity dispersion [6]. Size of grid step should be small enough to satisfy fast spatial field decay near metallic surface when localized plasmon resonance occurs, especially at the sharp corners. This leads to grid steps of about 0.001 of wavelength and causes great demands in memory and time consumption. The main goal of this work is to introduce simple model of surface roughness in FDTD which does not involve objects with complicated shapes and helps to reduce computational costs.

\section{Introducing of Surface Roughness in FDTD}

Two-dimensional spatial Fourier spectrum is a versatile approach for rough surfaces description because it is capable of precise transfer of experimentally measured roughness of given materials to the simulation program. Let define components of Fourier spectrum as $\mathrm{C}_{\mathrm{k}, \mathrm{p}}$ :

$$
\mathrm{C}_{\mathrm{k}, \mathrm{p}}=\sum_{\mathrm{j}=0}^{\mathrm{J}-1} \sum_{\mathrm{m}=0}^{\mathrm{M}-1} \mathrm{x}_{\mathrm{j}, \mathrm{m}} \mathrm{e}^{2 \pi \mathrm{i}(\mathrm{jk} / \mathrm{J}+\mathrm{pm} / \mathrm{M}),}
$$

where $\mathrm{J}, \mathrm{M}$ are lengths of an array in two dimensions; $\mathrm{x}_{\mathrm{j}, \mathrm{m}}$ is array of deviation of surface position from ideal contour. User definition of roughness spatial spectrum is also available, one can specify spectrum contour as superposition of Gauss-shape functions:

$$
\left|\mathrm{C}_{\mathrm{k}, \mathrm{p}}\right|=\frac{1}{2 \pi \delta_{\mathrm{k}} \delta_{\mathrm{p}}} \exp \left(-\frac{\left(\mathrm{k}-\omega_{\mathrm{k}}\right)^{2}}{2 \delta_{\mathrm{k}}^{2}}-\frac{\left(\mathrm{p}-\omega_{\mathrm{p}}\right)^{2}}{2 \delta_{\mathrm{p}}^{2}}\right),
$$

where $\delta_{\mathrm{k}}, \delta_{\mathrm{p}}$ are standard deviation, defining spectrum width for two dimensional face of a figure; $\omega_{\mathrm{k}}, \omega_{\mathrm{p}}$ are main spatial modes. Phase of spectrum components is chosen as random number:

$$
\mathrm{C}_{\mathrm{k}, \mathrm{p}}=\left|\mathrm{C}_{\mathrm{k}, \mathrm{p}}\right| \cdot \exp \left(2 \pi \mathrm{i} \varphi_{\mathrm{k}} \varphi_{\mathrm{p}}\right),
$$

where $\varphi_{\mathrm{k}}, \varphi_{\mathrm{p}}$ are random values with uniform distribution in a range from 0 to 1 . Maximum value of $C_{k, p}$ spectrum defines root mean square (RMS) of surface deviation, often 
referred to as line edge roughness (LER), which could be obtained by experimental measurements as follows:

$$
\sigma_{j}=\sqrt{\frac{\sum_{m} x_{j, m}^{2}}{M}} .
$$

Arbitrary shaped object in our program could be defined as a set of primitive figures: parallelepipeds, spheres, cylinders, hollow cylinders, spherical sectors, truncated pyramids. The surface of geometrical primitive is divided in several faces, each face can have its own roughness parameters, which consist of value of LER and components of two-dimensional complex spatial spectrum.

Several types of rough surfaces with different spatial spectrum are considered in simulation below. Examples of surface realization are showed in Fig. 1. Surface roughness is introduces at the top and bottom surface $((\mathrm{x}, \mathrm{y})$ plane $)$ of Ag layer, covered by $\mathrm{TiO}_{2}$ layers from both sides. Here spatial spectrum is assumed to be symmetrical with $\sigma=\sigma_{x}=\sigma_{y}, \delta=\delta_{x}=\delta_{y}, \omega=\omega_{x}=\omega_{y}$. The boundary conditions of FDTD in $\mathrm{x}$ - and $\mathrm{y}$-directions are periodic, and in $\mathrm{z}$-direction perfectly absorbing boundary condition (convolutional perfectly matched layer, C-PML) is placed.
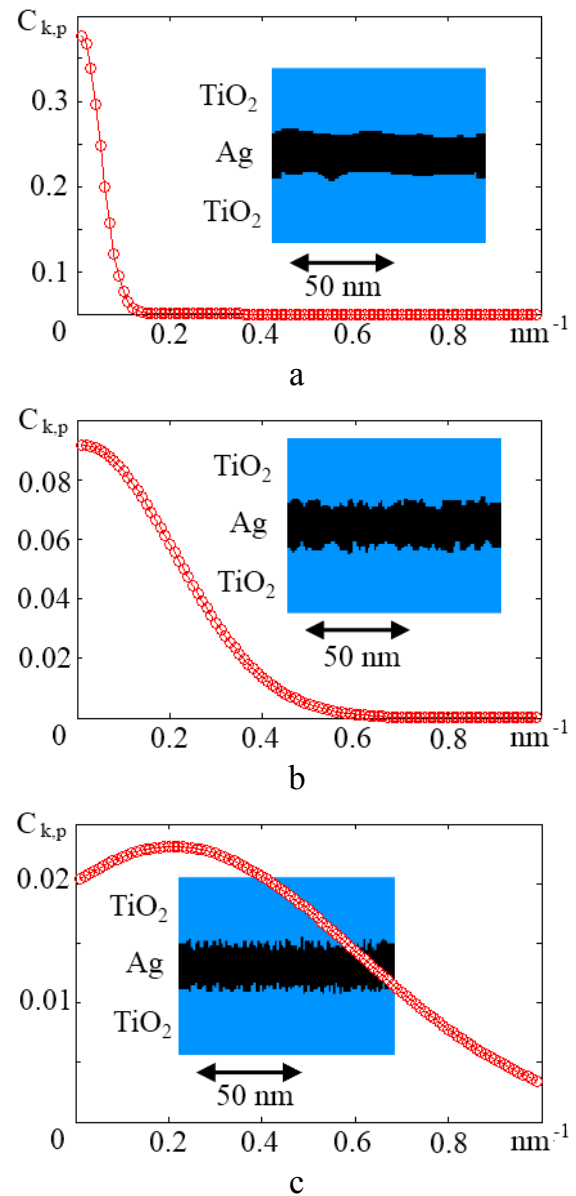

Figure 1: Examples surface profile realizations for different parameters of surface roughness of Ag layer, $\sigma=1 \mathrm{~nm}, \delta=0.04 \mathrm{~nm}^{-1}, \omega=0.0 \mathrm{~nm}^{-1}(\mathrm{a}) ; \sigma=1 \mathrm{~nm}, \delta=0.2$ $\mathrm{nm}^{-1}, \omega=0.0 \mathrm{~nm}^{-1}(\mathrm{~b}) ; \sigma=1 \mathrm{~nm}, \delta=0.4 \mathrm{~nm}^{-1}, \omega=0.2 \mathrm{~nm}^{-1}$ (c)

\section{Method of obtaining of effective parameters for roughness model}

Frequently used model of surface roughness considers replacement of roughness by intermediate layers with thickness depending on value of LER and refractive index averaged over indices of neighboring layers. This model comes from ellipsometry [7]. We failed to employ this approach for our test structures, since it did not provide reflection and transmission coefficients coincided with FDTD simulated results for rough surfaces. Possibly, it is not suitable for layers with thickness of about $30 \mathrm{~nm}$. As a result of our investigation we concluded that the proper model of surface roughness is to modify refractive index of dielectric layers in metal-dielectric stratified medium. Appropriate index values could be found by inverse problem solving. FDTD simulation of reflection and transmission coefficients of the structure with surface roughness gives results of direct problem solving. Modified values of dielectric layers refractive index pass to transfer matrix method which gives reflectance and transmittance that are compared to coefficients simulated with FDTD. Thus, values of refractive index of dielectric layers are found by minimizing target function:

$$
f(a, b)=|r-a|+|t-b|,
$$

where $a, b$ are reflection and transmission coefficients calculated by transfer matrix method for multi-layered structure; $r, t$ are reflection and transmission coefficients calculated by FDTD for definite profile of the structure with rough surface. Conjugate gradient method for nonlinear equations was used for minimizing of function (5).

\section{Test structures description}

Parameters of test structures were chosen according to design of flat lens with hyperbolic dispersion, described in [8].

Surface roughness in test structures was set at silver layers while titanium dioxide layers were considered flat having modified refractive index. Test structures examined below were consisted of either 3 or 5 layers. Number of rough layers occurred to be important parameter that affects transmittance of multilayered structures.

Three-layered test structure consists of $20 \mathrm{~nm} \mathrm{Ag} \mathrm{layer}$ surrounded by $30 \mathrm{~nm} \mathrm{TiO}_{2}$ layers (Fig. 1) with air above and below. Length of the computational domain in both directions parallel to the surface is taken $100 \mathrm{~nm}$. Periodic boundary conditions assume infinite duplication of the test structure along $\mathrm{x}$ and $\mathrm{y}$ axes parallel to the layer interfaces. Three different numerical simulations for obtaining real (n) and imaginary $(\mathrm{k})$ part of titanium dioxide refractive index were performed in FDTD for each set of roughness parameters. The structure was illuminated by normal incident plane wave with the wavelength of $358 \mathrm{~nm}$. Comparing results of amplitude of reflection $(|\mathrm{r}|)$ and transmission $(|\mathrm{t}|)$ coefficients one can estimate repeatability of parameters restoration for different realization of metal surface with the same roughness. Value of target function (f) was shown for understanding convergence of inverse 
problem solving, magnitude of target function corresponds to difference between amplitude coefficients calculated by transfer matrix method and by FDTD (Table 1).

Table 1: Effective parameters evaluation for threelayered structure.

\begin{tabular}{|c|c|c|c|c|c|}
\hline $\begin{array}{c}\text { roughness } \\
\text { parameters }\end{array}$ & $|\mathrm{r}|$ & $|\mathrm{t}|$ & $\mathrm{n}\left(\mathrm{TiO}_{2}\right)$ & $\mathrm{k}\left(\mathrm{TiO}_{2}\right)$ & $\begin{array}{c}\mathrm{f} \\
{\left[10^{-4}\right]}\end{array}$ \\
\hline$\omega=0 \mathrm{~nm}^{-1}$ & 0.552 & 0.76 & 2.5746 & 0.0181 & 8.7 \\
$\delta=0.04 \mathrm{~nm}^{-1}$ & 0.552 & 0.7625 & 2.5734 & 0.017 & 7.8 \\
$\sigma=2 \mathrm{~nm}^{-1}$ & 0.5571 & 0.7631 & 2.5768 & 0.0149 & 7 \\
\hline$\omega=0 \mathrm{~nm}^{-1}$ & 0.5184 & 0.7362 & 2.5632 & 0.0407 & 3 \\
$\delta=0.04 \mathrm{~nm}^{-1}$ & 0.5312 & 0.7342 & 2.5731 & 0.032 & 3.1 \\
$\sigma=4 \mathrm{~nm}^{-1}$ & 0.5112 & 0.7308 & 2.56 & 0.0457 & 8.7 \\
\hline$\omega=0 \mathrm{~nm}^{-1}$ & 0.5648 & 0.7636 & 2.582 & 0.0124 & 4 \\
$\delta=0.2 \mathrm{~nm}^{-1}$ & 0.5642 & 0.7637 & 2.582 & 0.0128 & 8.7 \\
$\sigma=0.5 \mathrm{~nm}^{-1}$ & 0.5649 & 0.7631 & 2.58 & 0.0126 & 3.7 \\
\hline$\omega=0 \mathrm{~nm}^{-1}$ & 0.5519 & 0.748 & 2.581 & 0.0242 & 0.36 \\
$\delta=0.2 \mathrm{~nm}^{-1}$ & 0.551 & 0.7471 & 2.5802 & 0.0243 & 1.3 \\
$\sigma=1 \mathrm{~nm}^{-1}$ & 0.5511 & 0.7482 & 2.5809 & 0.0249 & 1.16 \\
\hline$\omega=0.2 \mathrm{~nm}^{-1}$ & 0.562 & 0.7611 & 2.581 & 0.0143 & 11 \\
$\delta=0.4 \mathrm{~nm}^{-1}$ & 0.562 & 0.7612 & 2.5803 & 0.0143 & 8.8 \\
$\sigma=0.5 \mathrm{~nm}^{-1}$ & 0.5618 & 0.7614 & 2.5805 & 0.0143 & 10 \\
\hline$\omega=0.2 \mathrm{~nm}^{-1}$ & 0.5518 & 0.7494 & 2.58 & 0.0231 & 8 \\
$\delta=0.4 \mathrm{~nm}^{-1}$ & 0.5513 & 0.7489 & 2.579 & 0.0241 & 7 \\
$\sigma=1 \mathrm{~nm}$ & 0.5518 & 0.7494 & 2.58 & 0.0231 & 8 \\
\hline
\end{tabular}

Table 2: Effective parameters evaluation for five-layered structure.

\begin{tabular}{|c|c|c|c|c|c|}
\hline $\begin{array}{l}\text { roughness } \\
\text { parameters }\end{array}$ & $|\mathrm{r}|$ & $|t|$ & $\mathrm{n}\left(\mathrm{TiO}_{2}\right)$ & $\mathrm{k}\left(\mathrm{TiO}_{2}\right)$ & $\begin{array}{c}\mathrm{f} \\
{\left[10^{-4}\right]}\end{array}$ \\
\hline$\omega=0 \mathrm{~nm}^{-1}$ & 0.56 & 0.383 & 2.0807 & 0.0883 & 3.3 \\
\hline $\begin{array}{c}\delta=0.04 \mathrm{~nm}^{-1} \\
\sigma=0.5 \mathrm{~nm}\end{array}$ & 0.5595 & 0.3824 & 2.0808 & 0.0888 & 4.9 \\
\hline$\omega=0 \mathrm{~nm}^{-1}$ & 0.5522 & 0.3804 & 2.0641 & 0.0902 & 3.55 \\
\hline $\begin{array}{c}\delta=0.04 \mathrm{~nm}^{-1} \\
\sigma=1 \mathrm{~nm}\end{array}$ & 0.5740 & 0.3678 & 2.1083 & 0.0106 & 5.2 \\
\hline$\omega=0 \mathrm{~nm}^{-1}$ & 0.5803 & 0.3289 & 2.1119 & 0.1546 & 0.2 \\
\hline $\begin{array}{c}\delta=0.04 \mathrm{~nm}^{-1} \\
\sigma=2 \mathrm{~nm}\end{array}$ & 0.5368 & 0.3688 & 2.0269 & 0.0995 & 3.14 \\
\hline$\omega=0 \mathrm{~nm}^{-1}$ & 0.5963 & 0.3377 & 2.8708 & 0.14 & 8.6 \\
\hline $\begin{array}{c}\delta=0.04 \mathrm{~nm}^{-1} \\
\sigma=0.5 \mathrm{~nm}\end{array}$ & 0.5988 & 0.3376 & 2.8746 & 0.1387 & 8.1 \\
\hline$\omega=0 \mathrm{~nm}^{-1}$ & 0.5897 & 0.3029 & 2.1289 & 0.1924 & 4.5 \\
\hline $\begin{array}{c}\delta=0.2 \mathrm{~nm}^{-1} \\
\sigma=1 \mathrm{~nm}\end{array}$ & 0.5868 & 0.3049 & 2.1201 & 0.1886 & 2.8 \\
\hline$\omega=0 \mathrm{~nm}^{-1}$ & 0.5714 & 0.2511 & 1.9463 & 0.2476 & 1.6 \\
\hline $\begin{array}{c}\delta=0.2 \mathrm{~nm}^{-1} \\
\sigma=2 \mathrm{~nm}\end{array}$ & 0.572 & 0.2498 & 1.663 & 0.13 & 1.4 \\
\hline$\omega=0 \mathrm{~nm}^{-1}$ & 0.5577 & 0.1588 & no & & \\
\hline $\begin{array}{c}\delta=0.2 \mathrm{~nm}^{-1} \\
\sigma=4 \mathrm{~nm}\end{array}$ & 0.5587 & 0.1591 & result & & \\
\hline
\end{tabular}

Refractive index of titanium dioxide was considered 2.55 in FDTD calculations. According to Table 1, imaginary part of refractive index of dielectric layers rises proportionally to LER value for the surfaces with wide spatial spectrum. Reflection and transmission coefficients become dependent on random surface realization for the LER values more than $2 \mathrm{~nm}$.

Five layer test structure consists of following sequence: $32 \mathrm{~nm} \mathrm{Ag} \mathrm{layer,} 30 \mathrm{~nm} \mathrm{TiO}$ layer, $30 \mathrm{~nm} \mathrm{Ag} \mathrm{layer,} 30 \mathrm{~nm}$ $\mathrm{TiO}_{2}$ layer, $32 \mathrm{~nm} \mathrm{Ag}$ layer [8].

Amplitude coefficients for ideal smooth layers from FDTD calculations are $|\mathrm{r}|=0.5592,|\mathrm{t}|=0.5218$. According to Table 2 , scattering on rough surfaces of Ag significantly reduces transparency of the samples. Increase of number of layers from 3 to 5 produces worse convergence of effective parameters calculations. Different variants of surface distribution with narrow spatial spectrum and RMS $>1 \mathrm{~nm}$ show divergence in reflection and transmission coefficients calculation, while samples with wide spatial spectrum have similar coefficients when the same RMS is considered. On the other hand rising of RMS for wide spectrum roughness realizations produces results that do not match transfer matrix method and the model of effective index for roughness description is invalid (see table 2 for $\omega=0 \mathrm{~nm}^{-1}$, $\left.\delta=0.2 \mathrm{~nm}^{-1}, \sigma=4 \mathrm{~nm}\right)$.

\section{Simulation of flat lens with rough surfaces}

In this section we examine suitability of roughness model for description of propagation of diffracted light in flat lens. Fig. 2 presents ideal planar structure consisted of 5 layers with $\mathrm{TiO}_{2}$ refractive index of $n=2.55$. Linearly polarized light with wavelength of $\lambda=358 \mathrm{~nm}$ passes through $50 \mathrm{~nm}$ wide slit in $60 \mathrm{~nm}$ thick Ag layer. The distribution of amplitude of electric field after the last Ag layer is shown in contour plot normalized to the maximum of amplitude of incident wave.

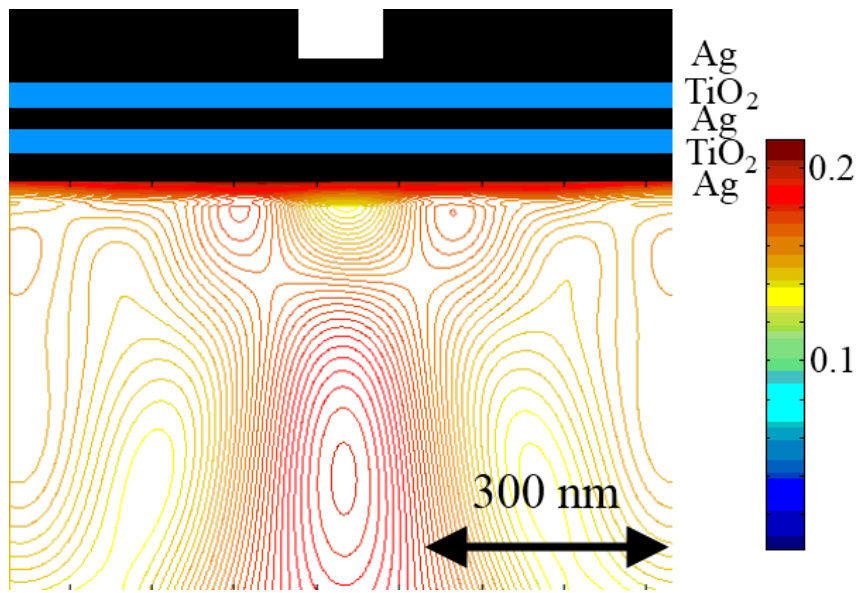

Figure 2: Diffraction of electric field after passing through hyperbolic metamaterial with flat surface.

According to effective medium theory, effective electric permittivity of composite material presented in Fig. 2, should be $\varepsilon_{\mathrm{x}}=\varepsilon_{\mathrm{y}}=1.8655+\mathrm{i} 0.2731 ; \quad \varepsilon_{\mathrm{z}}=-1.9629+\mathrm{i} 0.9177$; effective refractive index: $\mathrm{n}_{\mathrm{x}}=\mathrm{n}_{\mathrm{y}}=1.3695+\mathrm{i} 0.0997$; $\mathrm{n}_{\mathrm{z}}=0.3193+\mathrm{i} 1.4370$ (after parameters used in simulation: $\varepsilon(\mathrm{Ag})=-2.2745+\mathrm{i} 0.7214, \varepsilon\left(\mathrm{TiO}_{2}\right)=6.5025$, filling factor of $\mathrm{Ag} \eta=0.6104)$. Negative part of electric permittivity for wave components, travelling parallel to metal-dielectric 
interface, causes diffracted waves change direction at Agair interface. Thus small focusing effect is observed. In Fig. 3 surface roughness on Ag layers is placed. Roughness parameters for this case are $\omega=0 \mathrm{~nm}^{-1}, \delta=0.2 \mathrm{~nm}^{-1}, \sigma=1 \mathrm{~nm}$, which corresponds to modified refractive index of $\mathrm{n}\left(\mathrm{TiO}_{2}\right)=2.1201+\mathrm{i} 0.1886$ according to Table 2. This refractive index was substituted in calculation shown in Fig. 4.

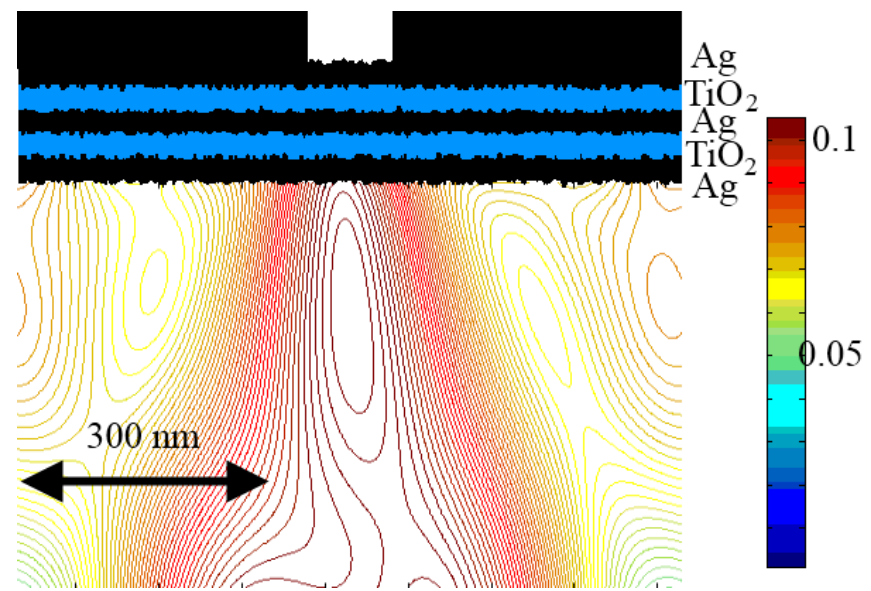

Figure 3: Distribution of module of electric field after passing through hyperbolic metamaterial with rough surface.

Effective electric permittivity of metal-dielectric slab in a case of refractive index of $\mathrm{TiO}_{2}$ taken from Table 2 should be $\varepsilon_{\mathrm{x}}=\varepsilon_{\mathrm{y}}=1.0695+\mathrm{i} 0.5847 ; \quad \varepsilon_{\mathrm{z}}=-1.9758+\mathrm{i} 1.0581 ; \quad \mathrm{n}_{\mathrm{x}}=\mathrm{n}_{\mathrm{y}}=$ $1.0697+\mathrm{i} 0.2733 ; \mathrm{n}_{\mathrm{z}}=0.3643+1.4521$. Small increase of imaginary part of refractive index is obtained. Presence of surface roughness gives similar diffracted field patterns and transmission coefficient as in the case of planar layers with modified refractive index. Asymmetry in Fig. 3 could explained by presence of random surface deviations, which have the size comparable to slit width.

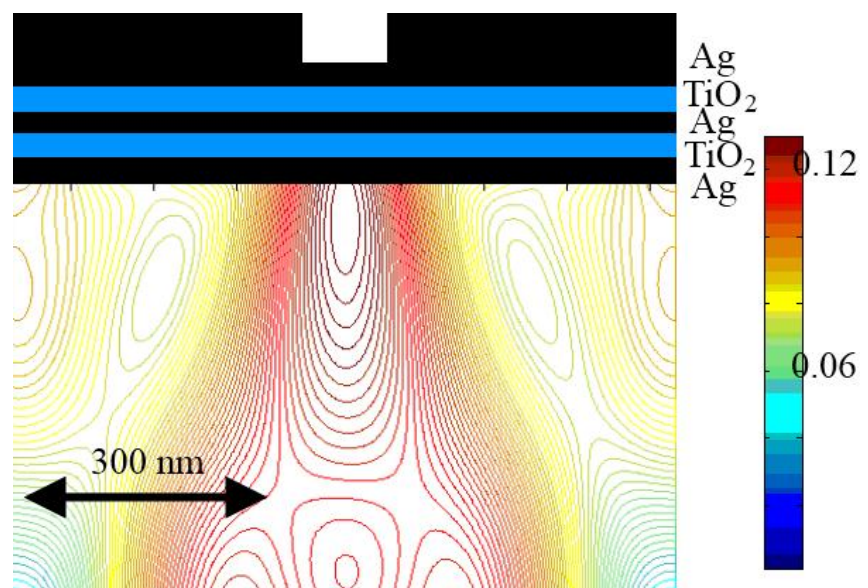

Figure 4: Distribution of module of electric field after passing through hyperbolic metamaterial with index of dielectric layers $\mathrm{n}\left(\mathrm{TiO}_{2}\right)=2.1201+\mathrm{i} 0.1886$.

\section{Conclusions}

We described and proved numerically that the influence of surface roughness at the interfaces in metal-dielectric composite materials could be described by proper selection of refractive index of dielectric layers. This approach is better than introducing additional intermediate layers or setting rigorous roughness profile in such grid method as FDTD, because it does not need fine grid resolution. It also allows employing simple and fast matrix transfer method for simulation of stratified structures with rough surfaces. However, understanding limits of application of this method of refractive index modification needs further study. Our calculation show, that some examples of roughness realization with narrow spatial spectrum and high LER value could not be described by results of transfer matrix method.

\section{Acknowledgements}

The authors would like to thank King Abdul-Aziz City for Science and Technology (KACST) for Research Grant No. 814-33.

\section{References}

[1] H.K. Yuan, U.K. Chettiar, W. Cai, A.V. Kildishev, A. Boltasseva, V.P. Drachev, V.M. Shalaev, A negative permeability material at red light, Optics Express 15: 1076-1083, 2007.

[2] S.E. Kozik, A.G. Smirnov, Full-scale simulation of angular-resolved focused-beam scatterometry applied to aperiodic isolated features: model validity analysis and numerical results, Proc. SPIE 8083: 80830A-180830A-13, 2011.

[3] A. Farjadpour, D. Roundy, A. Rodriguez, M. Ibanescu, P. Bermel, J. D. Joannopoulos, and S. G. Johnson, Improving accuracy by subpixel smoothing in the finitedifference time domain, Opt. Letters 31: 2972-2974, 2006

[4] A. Mohammadi, M. Agio, Dispersive contour-path finite-difference time-domain algorithm for modeling surface plasmon polaritons at flat interfaces, Optics Express 14: 11330-11338, 2006.

[5] Y. Zhao, P. Belov, Y. Hao, Accurate modelling of lefthanded metamaterials using a finite-difference timedomain method with spatial averaging at the boundaries, J. Opt. A: Pure Appl. Opt. 9: S468, 2007.

[6] A. Deinega, I. Valuev, Subpixel smoothing for conductive and dispersive media in the finite-difference time-domain method, Opt. Lett., 32: 3429-3431, 2007.

[7] H. Fujiwara, Spectroscopic Ellipsometry: Principles and Applications, Wiley, Chichester, 2007

[8] T. Xu, A. Agrawal, M. Abashin, K.J. Chau, H.J. Lezec, Allangle negative refraction and active flat lensing of ultraviolet light, Nature 497: 470-474, 2013 